\title{
Multigrid optimization methods for linear and bilinear elliptic optimal control problems*
}

\author{
$\begin{array}{ll}\text { M. } \text { VALLEJOS }^{\dagger} \quad \text { A. BORZI } & \\ & \end{array}$
}

\begin{abstract}
Multigrid optimization schemes that solve elliptic linear and bilinear optimal control problems are discussed. For the solution of these problems, the multigrid for optimization (MGOPT) method and the collective smoothing multigrid (CSMG) method are developed and compared. It is shown that thought these two methods are formally similar, they provide different computational properties.
\end{abstract}

Keywords: multigrid optimization methods.

AMS: 35Q40, 49K10, 65M06, 81V80.

\section{Introduction}

Although the multigrid strategy was first introduced to design solvers for elliptic boundary value problems, it is now considered as one of the most promising approaches for the development of efficient optimization schemes. Some recent developments include the application of one-shot multigrid schemes to optimality systems [1,5], to unconstrained optimization problems $[9,10,12]$, and to inverse problems $[15,16]$.

The purpose of this paper is to investigate two representative multigrid methods for optimization: the collective smoothing multigrid method (CSMG) and the multigrid for optimization method (MGOPT). In our investigation we consider the application of these methods for solving linear and bilinear elliptic optimal control problems. While both schemes are based on the well known full approximation storage (FAS) scheme [6] they represent different approaches to the solution of optimization problems. The CSMG scheme solves optimal control problems by solving the corresponding PDE optimality system in one shot treating all optimization variables collectively. As typical in multigrid development, this approach needs to customize the collective smoothing and intergrid transfer operators for each individual problem, i.e. the CSMG cannot be used as a black-box solver for all optimization problems. On the other hand, an appropriate design of the CSMG multigrid components results in a robust algorithm with typical multigrid efficiency. This fact is proved in [5] for

\footnotetext{
* Supported in part by the Austrian Science Fund FWF projects P18136-N13 "Quantum optimal control of semiconductor nanostructures" and F3205-N18 "Fast Multigrid Methods for Inverse Problems".

${ }^{\dagger}$ Institut für Mathematik und Wissenschaftliches Rechnen, Karl-Franzens-Universität Graz, Heinrichstr. 36, 8010 Graz, Austria (michelle.vallejoseuni-graz . at) and Institute of Mathematics, College of Science, University of the Philippines, Diliman, Quezon City, Philippines

${ }^{\ddagger}$ Institut für Mathematik und Wissenschaftliches Rechnen, Karl-Franzens-Universität Graz, Heinrichstr. 36, 8010 Graz, Austria (alfio.borzi@uni-graz.at).
} 
linear control problems. In this paper, we propose a CSMG method for bilinear control problems and use this scheme as a benchmark.

The motivation for investigating the MGOPT scheme $[10,12]$ is that it can be formulated in a way that is not problem specific and therefore it appears to have much easier and larger applicability. In the MGOPT scheme the multigrid solution process represents the outer loop where the control function is considered as the unique dependent variable. The inner loop in this scheme consists of a classical one-grid optimization scheme and the other MGOPT components are chosen as those typical of a classical multigrid approach. However, in contrast to the collective smoothing multigrid framework, we do not have yet a criteria for choosing the MGOPT components. In fact, as we show later, the essential condition for a 'successful' application of the MGOPT scheme is that the reduced Hessian, that is the Hessian of the optimization problem in the space of the control function, be positive definite. This seams a much less restrictive requirement than ellipticity of the constitutive equations of the optimality system which are required in the CSMG method. In this paper, we consider the MGOPT method applied to linear and bilinear elliptic optimal control problems and compare its numerical performance with that of the CSMG method. We investigate the influence of the choice of the one-grid optimization scheme on the MGOPT efficiency. Furthermore, also based on theoretical considerations, we investigate the robustness of the MGOPT scheme with respect to the choice of values of the optimization parameter.

In the following section, optimal control problems are formulated together with the first-order necessary optimality conditions and the second-order sufficient conditions for a minimum. In Section 3, we discuss two models for linear and bilinear distributed elliptic optimal control problems. We remark that the bilinear optimal control model is a much less investigated problem and it is similar to parameter identification problems. In Section 4 , smoothing schemes for the proposed elliptic optimal control problems is presented. In particular, we discuss a new robust collective smoothing scheme for optimal control problems with bilinear structure. The CSMG method and the MGOPT method are discussed in Section 5 and Section 6, respectively. We illustrate the similar structure of the two schemes even though the former applies to all dependent variables while the latter is formulated in the reduced space of the optimization variable. Convergence of the MGOPT method and results of numerical experiments follow to show the efficiency of both techniques and a conclusion completes this paper.

\section{Optimal control framework}

An optimal control problem governed by a partial differential equation (PDE) is formulated as follows

$$
\left\{\begin{array}{c}
\min _{u \in U} J(y, u) \\
c(y, u)=0
\end{array}\right.
$$

where $c(y, u)=0$ is a PDE that represents the equality constraint. We consider $c$ to be an elliptic PDE defined in a convex open $\Omega \subset \mathbb{R}^{2}$ with given boundary conditions. The state and the control (optimization) variables of the constraint $c$ are denoted by $y$ and $u$, respectively. We assume that, for a given $u$ the state equation admits a unique solution.

We consider a cost functional of the tracking type given by

$$
J(y, u)=h(y)+\nu g(u),
$$

where $\nu>0$ is the weight of the cost of the control. The functions $g$ and $h$ are required to be twice continuously differentiable, bounded from below and $g(u) \rightarrow \infty$ as $\|u\| \rightarrow \infty$. 
Given an optimization problem, the optimality system represents the first-order necessary conditions for a minimum. In order to derive these conditions, let $c: Y \times U \rightarrow Z$ for appropriate Hilbert spaces $Y, U$ and $Z$, and consider the following Lagrange functional

$$
L(y, u, p)=J(y, u)+\langle c(y, u), p\rangle_{Z, Z^{*}},
$$

where $p$ is the Lagrange multiplier, also known as the adjoint variable. By equating to zero the Frechét derivatives of $L$ with respect to the triple $(y, u, p)$, we have the optimality system

$$
\begin{aligned}
c(y, u) & =0, \\
h^{\prime}(y)+c_{y}(y, u)^{*} p & =0, \\
\nu g^{\prime}(u)+c_{u}(y, u)^{*} p & =0 .
\end{aligned}
$$

(* means adjoint.) The first differential equation in (3) is called the state equation and the second one is the adjoint equation. The last equation yields the optimality condition.

To better understand the importance of the last equation we introduce the reduced cost functional $\hat{J}$ given by

$$
\hat{J}(u)=J(y(u), u),
$$

where $y(u)$ denotes the unique solution to the state equation for a given $u$. One can show that the gradient of $\hat{J}(u)$ with respect to $u$ is given by

$$
\nabla \hat{J}(u)=\nu g^{\prime}(u)+c_{u}(y, u)^{*} p(u),
$$

where $p(u)$ solves the adjoint equation for a given $u$ and corresponding $y(u)$.

In a convex setting where the optimal control solution is unique, solving the optimality system is equivalent to solving the optimal control problem. However, in general, $c(y, u)=0$ may represent a nonlinear PDE and $g$ and $h$ may be locally non convex wherein problem (1) may have multiple extremals including minima, maxima, and even saddle points. Therefore additional conditions must be satisfied to guarantee that the solution is a minimizer. For the second-order optimality conditions, we assume that $(y, u, p)$ satisfy the optimality system (3) and the following

$$
L_{z z}(y, u, p)(v, v) \geq c_{1}\|v\|^{2}, \quad c_{1}>0 \quad \forall v \in \mathcal{N}\left(c^{\prime}(y, u)\right),
$$

where $z=(y, u)$ and $c^{\prime}(y, u)$ represents the linearized constraint. We assume that the null space $\mathcal{N}\left(c^{\prime}(y, u)\right)$ can be represented by $\mathcal{N}\left(c^{\prime}(y, u)\right)=T(y, u) U$, where $U$ is the space where the control is defined and

$$
T(y, u)=\left[\begin{array}{c}
-c_{y}^{-1} c_{u} \\
I_{u}
\end{array}\right],
$$

such that $c_{y}$ and $c_{u}$ are evaluated at $(y(u), u)$. Therefore, we can write condition (6) as

$$
\nabla^{2} \hat{J}(u)(w, w) \geq c_{2}\|w\|^{2} \quad c_{2}>0 \quad \forall w \in U .
$$

The operator $\nabla^{2} \hat{J}$ is the reduced Hessian defined by

$$
\nabla^{2} \hat{J}(u)=T^{*}(y, u) L_{z z}(y, u, p) T(y, u)
$$

where $y$ and $p$ solve the state and the adjoint equations for a given $u$. Hence, $\nabla^{2} \hat{J}(u)$ is given by

$$
\nabla^{2} \hat{J}=L_{u u}+C^{*} L_{y y} C-L_{u y} C-C^{*} L_{y u}
$$

where $C=C(y, u)=c_{y}^{-1}(y, u) c_{u}(y, u)$. Notice that the reduced Hessian matrix $\nabla^{2} \hat{J}$ is symmetric. Thus, condition (7) requires that the smallest real eigenvalue of the reduced Hessian be positive. 


\section{Linear and bilinear elliptic optimal control problems}

In this section, we discuss linear and a bilinear elliptic optimal control problems. In our setting the linear optimal control problem possesses a unique solution while for the bilinear case existence but no uniqueness of the optimal solution can be proved [11]. From an inverse problem point of view, the linear case represents a simplified source identification problem. On the other hand, the bilinear case belongs to the class of parameter identification problems.

We choose a cost functional given by

$$
J(y, u)=\frac{1}{2}\|y-z\|_{L^{2}(\Omega)}^{2}+\frac{\nu}{2}\|u\|_{L^{2}(\Omega)}^{2},
$$

where $z \in L^{2}(\Omega)$ is the target function. This choice corresponds to $h(y)=\frac{1}{2}\|y-z\|_{L^{2}(\Omega)}^{2}$ and $g(u)=\frac{1}{2}\|u\|_{L^{2}(\Omega)}^{2}$.

We focus on the following linear elliptic optimal control problem

$$
\left\{\begin{aligned}
\min _{u \in U} J(y, u), \\
-\Delta y-u=f \quad \text { in } \Omega, \\
y=0 \quad \text { on } \partial \Omega,
\end{aligned}\right.
$$

where $f \in L^{2}(\Omega)$ and $U=L^{2}(\Omega)$. In this case, $u$ is a distributed control over $\Omega$. The solution to (10) is characterized by the following optimality system

$$
\begin{array}{rll}
-\Delta y-u=f & & \text { in } \Omega, \\
y=0 & & \text { on } \partial \Omega, \\
-\Delta p+y=z & & \text { in } \Omega, \\
p=0 & & \text { on } \partial \Omega, \\
\nu u-p=0 & & \text { in } \Omega .
\end{array}
$$

From Equations (5) and (8), the reduced gradient is given by

$$
\nabla \hat{J}(u)=\nu u-p
$$

and the reduced Hessian is

$$
\nabla^{2} \hat{J}(u)=\nu \mathrm{I}+\Delta^{-2}
$$

We see that $\nabla^{2} \hat{J}(u)$ is strictly positive and its eigenvalues are given by

$$
\lambda_{m n}=\nu+\frac{1}{\pi^{4}\left(m^{2}+n^{2}\right)^{2}} \quad \text { for } m, n \in \mathbb{N}
$$

which are all positive. The smallest eigenvalue is $\lambda_{\min }=\nu>0$ and therefore the solution to the optimality system is guaranteed to be a minimizer. However, smaller $\nu$ correspond to more flat minima and more stiff optimality systems.

Before we discuss the bilinear case, we consider two conditions regarding the reduced Hessian which are needed in Section 7. These conditions are necessary for proving of convergence of the MGOPT scheme. First, we have the ellipticity condition given by

$$
\left(\nabla^{2} \hat{J}(u) v, v\right) \geq \beta\|v\|^{2} \quad \text { for some } \quad \beta>0 .
$$

We find an appropriate $\beta$ as follows

$$
\begin{aligned}
\left(\nabla^{2} \hat{J}(u) v, v\right) & =\left(\left(\nu I+\Delta^{-2}\right) v, v\right)=\nu(v, v)+\left(\Delta^{-2} v, v\right) \\
& \geq \nu(v, v)=\nu\|v\|^{2} .
\end{aligned}
$$


Hence $\beta=\nu$. Also we have the Lipschitz condition

$$
\left\|\nabla^{2} \hat{J}(u)-\nabla^{2} \hat{J}(v)\right\| \leq \lambda\|u-v\| \text { uniformly for some } \lambda \geq 0 .
$$

And it is easily shown that $\lambda=0$ since $\left\|\nabla^{2} \hat{J}(u)-\nabla^{2} \hat{J}(v)\right\|=0$.

Next, consider the case of a bilinear optimal control problem given by the following

$$
\left\{\begin{aligned}
\min _{u \in U} J(y, u), & \\
-\Delta y-u y=f & \text { in } \quad \Omega \\
y=0 & \text { on } \quad \partial \Omega .
\end{aligned}\right.
$$

Similar to the linear case, we choose $f \in L^{2}(\Omega)$ and $U=L^{2}(\Omega)$. The solution to problem (14) is characterized by the following optimality system

$$
\begin{aligned}
& -\Delta y-u y=f \quad \text { in } \Omega, \\
& y=0 \quad \text { on } \partial \Omega \text {, } \\
& -\Delta p+y-u p=z \quad \text { in } \Omega, \\
& p=0 \quad \text { on } \partial \Omega \text {, } \\
& \nu u-y p=0 \quad \text { in } \Omega \text {. }
\end{aligned}
$$

For a given $u, y(u)$ and $p(u)$ are the solutions of the state and adjoint equations with homogeneous Dirichlet boundary conditions. Their existence requires that the operator $(\Delta+u)$ be invertible. Now notice that $u$ cannot be constant on $\Omega$ since it satisfies $u=y p / \nu$ and it inherits the homogeneous boundary conditions as the state and the adjoint variables. Then we can use Lemma 3.2 from [8] based on the following theorem [7] that we state without proof.

Theorem 1 Let $\Omega \subset \mathbb{R}^{d}(d \geq 2)$ be open and connected, let $V \in L_{l o c}^{q}(\Omega)$ for some $q \geq 2$ with $q \geq \frac{2 d-1}{3}$. If $\psi \in H_{\text {loc }}^{1}(\Omega)$,

$$
(-\Delta+V) \psi=0
$$

and $\psi(x)=0$ on an open, non-empty subset of $\Omega$, then $\psi=0$ on $\Omega$.

We can now derive the reduced gradient and the reduced Hessian using (5) and (8) as follows

$$
\nabla \hat{J}(u)=\nu u-y p
$$

and

$$
\nabla^{2} \hat{J}(u)=\nu \mathrm{I}+y(\Delta+u)^{-2} y+p(\Delta+u)^{-1} y+y(\Delta+u)^{-1} p .
$$

In this case, we cannot state positivity of the reduced Hessian. And it is difficult to find the ellipticity and Lipschitz constants unlike in the linear case. However, since $p=(\Delta+$ $u)^{-1}(y-z)$ we can expect that for sufficiently accurate tracking, i.e. small $\|y-z\|$, and moderate values of $\nu$ the reduced Hessian is a positive definite operator. This situation may take place whenever $z$ is (almost) attainable. That is, there exists a $u$ such that $y(u) \approx z$.

\section{Discretization and collective smoothing}

We discuss multigrid methods for optimization problems that are formally expressed by set of equations $A u=f$ in a domain $\Omega$. Correspondingly, we need to define a hierarchy of problems $A_{k} u_{k}=f_{k}$, indexed by $k=1,2, \ldots, L$. These problems are assumed to be defined in $\Omega_{k}$ that represents $\Omega$ with a discretization parameterized by grid size $h_{k}$. We have 
$h_{1}>h_{2}>\cdots>h_{L}>0$, and for simplicity we assume that $h_{k-1}=2 h_{k}$. The number of interior grid points will be $n_{k}$, and any function in $\Omega_{k}$ is a vector of size $n_{k}$. We denote this vector space with $V_{k}$. In the space $V_{k}$, we introduce the discrete $L^{2}$ inner product $(\cdot, \cdot)_{k}$ with the corresponding norm $\|u\|_{k}=\sqrt{(u, u)_{k}}$.

For multigrid purpose we define a restriction operator $I_{k}^{k-1}: V_{k} \rightarrow V_{k-1}$ and a prolongation operator $I_{k-1}^{k}: V_{k-1} \rightarrow V_{k}$. We require that they satisfy $\left(I_{k}^{k-1} u, v\right)_{k-1}=$ $\left(u, I_{k-1}^{k} v\right)_{k}$ for all $u \in V_{k}$ and $v \in V_{k-1}$. That is, the restriction operator is the adjoint of the interpolation operator.

Now we consider the discrete version of the optimality system (11) for the linear control case. Let us consider finite difference discretization and $-\Delta_{k}$ denotes the minus five-point Laplacian. We have

$$
\begin{aligned}
-\Delta_{k} y_{k}-u_{k} & =f_{k}, \\
-\Delta_{k} p_{k}+y_{k} & =z_{k}, \\
\nu u_{k}-p_{k} & =0 .
\end{aligned}
$$

Let $x \in \Omega_{k}$ where $x=\left(i h_{k}, j h_{k}\right)$ and $i, j$ are the indeces of the grid points arranged lexicographically. Hence, in expanded form we have

$$
\begin{aligned}
-\left(y_{i-1, j}+y_{i+1, j}+y_{i, j-1}+y_{i, j+1}\right)+4 y_{i, j}-h^{2} u_{i, j} & =h^{2} f_{i, j}, \\
-\left(p_{i-1, j}+p_{i+1, j}+p_{i, j-1}+p_{i, j+1}\right)+4 p_{i, j}+h^{2} y_{i, j} & =h^{2} z_{i, j}, \\
\nu u_{i, j}-p_{i, j} & =0 .
\end{aligned}
$$

Let $w_{k}=\left(y_{k}, u_{k}, p_{k}\right)$. A collective smoothing step on $w$ updates the values $y_{i, j}, p_{i, j}$, and $u_{i, j}$ such that the resulting residuals of the state and adjoint equations at that point are zero. We first set

$$
\begin{aligned}
& A_{i, j}=-\left(y_{i-1, j}+y_{i+1, j}+y_{i, j-1}+y_{i, j+1}\right)-h^{2} f_{i, j}, \quad \text { and } \\
& B_{i, j}=-\left(p_{i-1, j}+p_{i+1, j}+p_{i, j-1}+p_{i, j+1}\right)-h^{2} z_{i, j} .
\end{aligned}
$$

The values $A_{i, j}$ and $B_{i, j}$ are considered constant during the update of the variables at $i j$. Hence, we have the following system of equations of three variables $y_{i, j}, u_{i, j}$ and $p_{i, j}$

$$
\begin{aligned}
A_{i, j}+4 y_{i, j}-h^{2} u_{i, j} & =0, \\
B_{i, j}+4 p_{i, j}+h^{2} y_{i, j} & =0, \\
\nu u_{i, j}-p_{i, j} & =0 .
\end{aligned}
$$

Since this is a linear system, we can compute the updates for the variables $y_{i, j}, u_{i, j}$ and $p_{i, j}$ in the following way

$$
\begin{aligned}
& y_{i, j}\left(u_{i, j}\right)=\frac{1}{4}\left(h^{2} u_{i, j}-A_{i, j}\right) \\
& p_{i, j}\left(u_{i, j}\right)=\frac{1}{16}\left(-h^{4} u_{i, j}+h^{2} A_{i, j}-4 B_{i, j}\right) .
\end{aligned}
$$

To obtain an update $u_{i, j}$, we require that it satisfies the optimality condition $\nabla \hat{J}(u)=$ $\nu u-p(u)=0$. Hence, we have

$$
u_{i, j}=\frac{1}{16 \nu+h^{4}}\left(h^{2} A_{i, j}-4 B_{i, j}\right) .
$$

With this $u_{i, j}$ we use (20) to update the values of the state and adjoint variable at the $i, j$ grid point. A sweep of this smoothing scheme consists in an ordered sequential update of $u_{i, j}$ and $y_{i, j}, p_{i, j}$ on all grid points.

Next, we define the smoothing iteration for the discrete bilinear elliptic optimal control problem given by

$$
\begin{aligned}
-\Delta_{k} y_{k}-u_{k} y_{k} & =f_{k}, \\
-\Delta_{k} p_{k}+y_{k}-u_{k} p_{k} & =z_{k}, \\
\nu u_{k}-y_{k} p_{k} & =0 .
\end{aligned}
$$


In this case, we have

$$
\begin{aligned}
-\left(y_{i-1, j}+y_{i+1, j}+y_{i, j-1}+y_{i, j+1}\right)+4 y_{i, j}-h^{2} u_{i, j} y_{i, j} & =h^{2} f_{i, j}, \\
-\left(p_{i-1, j}+p_{i+1, j}+p_{i, j-1}+p_{i, j+1}\right)+4 p_{i, j}+h^{2} y_{i, j}-h^{2} u_{i, j} p_{i, j} & =h^{2} z_{i, j}, \\
\nu u_{i, j}-y_{i, j} p_{i, j} & =0 .
\end{aligned}
$$

Using the same notations as in (19), $A_{i, j}$ and $B_{i, j}$ are considered constant during the update of the variables at $i j$. Hence, we have the following system of equations of three variables $y_{i, j}, u_{i, j}$, and $p_{i, j}$

$$
\begin{aligned}
A_{i, j}+4 y_{i, j}-h^{2} u_{i, j} y_{i, j} & =0, \\
B_{i, j}+4 p_{i, j}+h^{2} y_{i, j}-h^{2} u_{i, j} p_{i, j} & =0, \\
\nu u_{i, j}-y_{i, j} p_{i, j} & =0 .
\end{aligned}
$$

We see that the resulting system of equations is nonlinear and thus computing the updates for the variables $u_{i, j}, y_{i, j}$ and $p_{i, j}$ requires to apply a local Newton step. This approach results in a non robust smoothing iteration apparently because we have multiple solutions for $u_{i, j}$ that are close. In fact, as we show below, the condition $\nu u_{i, j}-y_{i, j}\left(u_{i, j}\right) p_{i, j}\left(u_{i, j}\right)=0$ results in a quartic polynomial equation for $u_{i, j}$ and therefore four roots are possible. To determine these solutions we construct the quartic polynomial and solve it exactly by using the Cardano-Tartaglia formula. In this way we can explore among the possible solutions of the optimization step. To construct the quartic polynomial, we can define $y_{i, j}=y_{i, j}\left(u_{i, j}\right)$ and $p_{i, j}=p_{i, j}\left(u_{i, j}\right)$ as functions of $u_{i, j}$ as follows

$$
\begin{aligned}
& y_{i, j}\left(u_{i, j}\right)=\frac{-1}{4-h^{2} u_{i, j}} A_{i, j}, \\
& p_{i, j}\left(u_{i, j}\right)=\frac{1}{\left(4-h^{2} u_{i, j}\right)^{2}}\left(h^{2} A_{i, j}+h^{2} B_{i, j} u_{i, j}-4 B_{i, j}\right),
\end{aligned}
$$

and equate the reduced gradient to zero, i.e. $\nabla \hat{J}(u)=\nu u-y(u) p(u)=0$. Hence we have a quartic polynomial equation in $u_{i, j}$ given by

$$
\nu h^{6} u_{i, j}^{4}-12 \nu h^{4} u_{i, j}^{3}+48 \nu h^{2} u_{i, j}^{2}-\left(64 \nu+h^{2} A_{i, j} B_{i, j}\right) u_{i, j}-\left(h^{2} A_{i, j}^{2}-4 A_{i, j} B_{i, j}\right)=0 .
$$

The solutions of the quartic polynomial are either four real or two real and two complex. The two complex conjugate solutions can be disregarded. In order to find the minimizer, we choose the minimum real solution of the quartic polynomial which minimizes

$$
\hat{J}_{i, j}(u)=\frac{1}{2}\left(y_{i, j}(u)-z_{i, j}\right)^{2}+\frac{\nu}{2} u^{2} .
$$

With this condition, we get a robust and efficient smoothing iteration.

\section{The collective smoothing multigrid (CSMG) method}

The CSMG scheme is based on the nonlinear multigrid full approximation storage (FAS) method applied to the optimality system with a collective smoothing. This method shows mesh independence due to its robustness with respect to the value of the weight of the control. Some recent applications of the CSMG method to linear control problems with state and control constraints are presented in [2,4]. To illustrate the CSMG method consider

$$
A_{k}\left(w_{k}\right)=f_{k},
$$

where $A_{k}(\cdot)$ represents the discrete optimality system and $w_{k}=\left(y_{k}, u_{k}, p_{k}\right)$. Let the collective smoothing iteration be denoted by $S_{k}$ such that we get an update $w_{k}^{l}=S_{k}\left(w_{k}^{l-1}, f_{k}\right)$. 
Starting with an initial approximation $w_{k}^{0}$, we apply $\gamma_{1}$ times the smoothing scheme and obtain $w_{k}^{\gamma_{1}}$. Now, the desired solution $w_{k}$ can be written as $w_{k}=w_{k}^{\gamma_{1}}+e_{k}$ for some error $e_{k}$ which is assumed to be smooth. We also have the residual $r_{k}=f_{k}-A_{k}\left(w_{k}^{\gamma_{1}}\right)$ associated with $w_{k}^{\gamma_{1}}$. Thus, the error $e_{k}$ can be viewed as the solution to the following equation

$$
A_{k}\left(w_{k}^{\gamma_{1}}+e_{k}\right)=r_{k}+A_{k}\left(w_{k}^{\gamma_{1}}\right) .
$$

Next, we want to solve problem (23) on a coarser grid $\Omega_{k-1}$. Define $w_{k-1}:=I_{k}^{k-1} w_{k}^{\gamma_{1}}+$ $e_{k-1}$. Here, $w_{k-1}$ represents a coarse-grid approximation to $w_{k}$. To determine $w_{k-1}$ we define a coarse-grid representation of $A_{k}(\cdot)$ by $A_{k-1}(\cdot)$, we approximate $w_{k}^{\gamma_{1}}$ by $I_{k}^{k-1} w_{k}^{\gamma_{1}}$ and $I_{k}^{k-1} r_{k}=I_{k}^{k-1}\left(f_{k}-A_{k}\left(w_{k}^{\gamma_{1}}\right)\right)$. Hence, we have the following equation

$$
A_{k-1}\left(w_{k-1}\right)=I_{k}^{k-1}\left(f_{k}-A_{k}\left(w_{k}^{\gamma_{1}}\right)\right)+A_{k-1}\left(I_{k}^{k-1} w_{k}^{\gamma_{1}}\right) .
$$

We define

$$
\tau_{k-1}=A_{k-1}\left(I_{k}^{k-1} w_{k}^{\gamma_{1}}\right)-I_{k}^{k-1} A_{k}\left(w_{k}^{\gamma_{1}}\right),
$$

then equation (24) can be written as

$$
A_{k-1}\left(w_{k-1}\right)=I_{k}^{k-1} f_{k}+\tau_{k-1} .
$$

The term $\tau_{k-1}$ is called the fine-to-coarse residual correction. The solution of equation (24) provides $e_{k-1}:=w_{k-1}-I_{k}^{k-1} w_{k}^{\gamma_{1}}$. Therefore, we have a correction to the fine grid approximation as follows

$$
w_{k}=w_{k}^{\gamma_{1}}+I_{k-1}^{k}\left(w_{k-1}-I_{k}^{k-1} w_{k}^{\gamma_{1}}\right) .
$$

Finally, we apply $\gamma_{2}$ iterations of the smoothing algorithm to damp possible high frequency errors that may arise from the coarse grid correction process. The following algorithm presents the method described above.

Algorithm 2 (CSMG algorithm) Initialize $w_{k}^{0}$ to be an initial approximation at resolution $k$. If $k=1$ (coarsest resolution), solve $A_{k}\left(w_{k}\right)=f_{k}$ and return. Else if $k>1$,

1. Pre-smoothing

Apply $\gamma_{1}$ iterations of an optimization algorithm to the problem at resolution $k$.

$$
w_{k}^{l}=S_{k}\left(w_{k}^{l-1}, f_{k}\right), \quad l=1,2, \ldots, \gamma_{1}
$$

2. Coarse grid problem

Compute the residual. $r_{k}=f_{k}-A_{k}\left(w_{k}^{\gamma_{1}}\right)$

Restrict the residual. $r_{k-1}=I_{k}^{k-1} r_{k}$

Restrict the solution of (1). $w_{k-1}^{\gamma_{1}}=I_{k}^{k-1} w_{k}^{\gamma_{1}}$.

Compute the fine-to-coarse residual correction.

$$
\tau_{k-1}=A_{k-1}\left(w_{k-1}^{\gamma_{1}}\right)-I_{k}^{k-1} A_{k}\left(w_{k}^{\gamma_{1}}\right), \quad f_{k-1}=I_{k}^{k-1} f_{k}+\tau_{k-1}
$$

Apply $\gamma$ cycles of $\operatorname{CSMG}\left(\gamma_{1}, \gamma_{2}\right)$ to the coarse grid problem $A_{k-1}\left(w_{k-1}\right)=f_{k-1}$ to obtain $w_{k-1}$

3. Coarse grid correction

$\overline{\text { Prolongate the error. } e}=I_{k-1}^{k}\left(w_{k-1}-w_{k-1}^{\gamma_{1}}\right)$

The coarse grid correction is given by $w_{k}^{\gamma_{1}+1}=w_{k}^{\gamma_{1}}+e$.

4. Post-smoothing

Apply $\gamma_{2}$ iterations of a smoothing algorithm to the problem at resolution $k$.

$$
u_{k}^{l}=S_{k}\left(w_{k}^{l-1}, f_{k}\right), \quad l=\gamma_{1}+2, \ldots, \gamma_{1}+\gamma_{2}+1
$$




\section{The multigrid for optimization (MGOPT) method}

The multigrid for optimization (MGOPT) method was first introduced by Nash [12] and Lewis and Nash $[9,10]$ as an extension of the multigrid scheme to optimization problems. To illustrate the MGOPT method, consider a discrete (locally) convex optimization problem

$$
\min _{u_{k}}\left(\hat{J}_{k}\left(u_{k}\right)-\left(f_{k}, u_{k}\right)_{k}\right) \text {. }
$$

This problem is equivalent to solving

$$
\nabla \hat{J}_{k}\left(u_{k}\right)=f_{k}
$$

We introduce the term $f_{k}$ in order to give a recursive formulation of the MGOPT scheme. At finest resolution $k=L$ we set $f_{k}=0$.

Our focus is on optimal control problems where $\hat{J}_{k}$ represents the reduced cost functional. To evaluate $\hat{J}_{k}$ at $u_{k}$ we compute $y_{k}\left(u_{k}\right)$ and to determine $\nabla \hat{J}_{k}\left(u_{k}\right)$ we need $y_{k}\left(u_{k}\right)$ and $p_{k}\left(u_{k}\right)$. The MGOPT scheme is formulated in terms of the functional and its gradient that implies the (formally exact) solution of the state and adjoint equations.

Let $S_{k}$ be an optimization algorithm on the space $V_{k}$ such that $u_{k}^{l}=S_{k}\left(u_{k}^{l-1}\right)$ for $l=1,2, \ldots, \gamma_{1}$. We require sufficient decrease as follows

$\hat{J}_{k}\left(u_{k}^{l}\right)-\left(f_{k}, u_{k}^{l}\right)_{k}<\hat{J}_{k}\left(u_{k}^{l-1}\right)-\left(f_{k}, u_{k}^{l-1}\right)_{k}-\eta\left\|\nabla \hat{J}_{k}\left(u_{k}^{l-1}\right)-f_{k}\right\|^{2} \quad$ for some $\eta \in(0,1)$.

Let $u_{k}^{0}$ be an initial approximation to the solution of Equation (28). After an application of $\gamma_{1}$ iterations of an optimization algorithm, we obtain $u_{k}^{\gamma_{1}}$. Hence, it follows that the desired solution $u_{k}$ is given by $u_{k}=u_{k}^{\gamma_{1}}+e_{k}$ for some error $e_{k}$. Therefore, problem (29) can be written as

$$
\nabla \hat{J}_{k}\left(u_{k}^{\gamma_{1}}+e_{k}\right)=f_{k}
$$

or equivalently as

$$
\nabla \hat{J}_{k}\left(u_{k}^{\gamma_{1}}+e_{k}\right)-\nabla \hat{J}_{k}\left(u_{k}^{\gamma_{1}}\right)=f_{k}-\nabla \hat{J}_{k}\left(u_{k}^{\gamma_{1}}\right) .
$$

Next, we will represent problem (30) on a coarser grid $V_{k-1}$. We define $u_{k}^{\gamma_{1}}+e_{k}$ on $V_{k-1}$ as

$$
u_{k-1}=I_{k}^{k-1} u_{k}^{\gamma_{1}}+e_{k-1} .
$$

On the left hand side of equation (30), we represent $\nabla \hat{J}_{k}(\cdot)$ by $\nabla \hat{J}_{k-1}(\cdot)$ and $u_{k}^{\gamma_{1}}$ by $I_{k}^{k-1} u_{k}^{\gamma_{1}}$. On the other side, we apply the restriction operator $I_{k}^{k-1}$ and we get $I_{k}^{k-1}\left(f_{k}-\right.$ $\left.\nabla \hat{J}_{k}\left(u_{k}^{\gamma_{1}}\right)\right)$. Hence, we have the following equation

$$
\nabla \hat{J}_{k-1}\left(u_{k-1}\right)=I_{k}^{k-1} f_{k}-I_{k}^{k-1} \nabla \hat{J}_{k}\left(u_{k}^{\gamma_{1}}\right)+\nabla \hat{J}_{k-1}\left(I_{k}^{k-1} u_{k}^{\gamma_{1}}\right) .
$$

If we represent $\tau_{k-1}$ as

$$
\tau_{k-1}=\nabla \hat{J}_{k-1}\left(I_{k}^{k-1} u_{k}^{\gamma_{1}}\right)-I_{k}^{k-1} \nabla \hat{J}_{k}\left(u_{k}^{\gamma_{1}}\right),
$$

then equation (32) can be written as

$$
\nabla \hat{J}_{k-1}\left(u_{k-1}\right)=I_{k}^{k-1} f_{k}+\tau_{k-1} .
$$

The term $\tau_{k-1}$ is called the fine-to-coarse gradient correction. Solving equation (34) is the same as solving the coarse-grid optimization problem

$$
\min _{u_{k-1}}\left(\hat{J}_{k-1}\left(u_{k-1}\right)-\left(f_{k-1}, u_{k-1}\right)_{k-1}\right)
$$


where $f_{k-1}=I_{k}^{k-1} f_{k}+\tau_{k-1}$. Assume that the solution to (35) is $u_{k-1}$. Then we can define the coarse-to-fine minimization step as follows

$$
u_{k}=u_{k}^{\gamma_{1}}+\alpha I_{k-1}^{k}\left(u_{k-1}-I_{k}^{k-1} u_{k}^{\gamma_{1}}\right) .
$$

The term $\alpha$ is the step length obtained after a line search procedure in the direction given by $I_{k-1}^{k}\left(u_{k-1}-I_{k}^{k-1} u_{k}^{\gamma_{1}}\right)$. Finally, we apply $\gamma_{2}$ iterations of the optimization algorithm and we get the solution $u_{k}^{\gamma_{1}+\gamma_{2}+1}$.

Remark 3 Let us consider the coarse grid optimization problem given in equation (35). Denote $u_{k-1}=I_{k}^{k-1} u_{k}$, then the gradient of the coarse grid functional is

$$
\begin{aligned}
\nabla\left(\hat{J}_{k-1}\left(u_{k-1}\right)\right. & \left.-\left(f_{k-1}, u_{k-1}\right)_{k-1}\right) \\
& =\nabla \hat{J}_{k-1}\left(u_{k-1}\right)-f_{k-1} \\
& =\nabla \hat{J}_{k-1}\left(u_{k-1}\right)-\left[I_{k}^{k-1} f_{k}+\nabla \hat{J}_{k-1}\left(u_{k-1}\right)-I_{k}^{k-1} \nabla \hat{J}_{k}\left(u_{k}\right)\right] \\
& =I_{k}^{k-1} \nabla \hat{J}_{k}\left(u_{k}\right)-I_{k}^{k-1} f_{k} \\
& =I_{k}^{k-1}\left(\nabla \hat{J}_{k}\left(u_{k}\right)-f_{k}\right) .
\end{aligned}
$$

Note that in the second equality, we use the definition of $f_{k-1}$ and $\tau_{k-1}$. This shows that the gradient of the coarse grid functional at $u_{k-1}$ is the same as the restriction of the gradient of the fine grid functional at $u_{k}$.

We are now ready to present one cycle of the iterative MGOPT method.

Algorithm 4 (MGOPT algorithm) Initialize $u_{k}^{0}$ to be an initial approximation at resolution $k$. If $k=1$ (coarsest resolution), solve $\min _{u_{k}}\left(\hat{J}_{k}\left(u_{k}\right)-\left(f_{k}, u_{k}\right)_{k}\right)$ and return, i.e., solve $\nabla \hat{J}_{k}\left(u_{k}\right)=f_{k}$. Else if $k>1$,

1. Pre-optimization

Apply $\gamma_{1}$ iterations of an optimization algorithm to the problem at resolution $k$.

$$
u_{k}^{l}=S_{k}\left(u_{k}^{l-1}\right), \quad l=1,2, \ldots, \gamma_{1}
$$

2. Coarse grid problem

Restrict the solution of (1). $u_{k-1}^{\gamma_{1}}=I_{k}^{k-1} u_{k}^{\gamma_{1}}$

Compute the fine-to-coarse gradient correction.

$$
\tau_{k-1}=\nabla \hat{J}_{k-1}\left(u_{k-1}^{\gamma_{1}}\right)-I_{k}^{k-1} \nabla \hat{J}_{k}\left(u_{k}^{\gamma_{1}}\right), \quad f_{k-1}=I_{k}^{k-1} f_{k}+\tau_{k-1}
$$

Apply one cycle of MGOPT $\left(\gamma_{1}, \gamma_{2}\right)$ to the coarse grid minimization problem

$$
\min _{u_{k-1}}\left(\hat{J}_{k-1}\left(u_{k-1}\right)-\left(f_{k-1}, u_{k-1}\right)_{k-1}\right)
$$

to obtain $u_{k-1}$

3. Coarse-to-fine minimization

Prolongate the error. $e=I_{k-1}^{k}\left(u_{k-1}-u_{k-1}^{\gamma_{1}}\right)$

Perform a line search in the direction e to obtain a step length $\alpha_{k}$.

The coarse-to-fine minimization step is given by $u_{k}^{\gamma_{1}+1}=u_{k}^{\gamma_{1}}+\alpha_{k}$ e.

4. Post-optimization

Apply $\gamma_{2}$ iterations of an optimization algorithm to the problem at resolution $k$.

$$
u_{k}^{l}=S_{k}\left(u_{k}^{l-1}\right), \quad l=\gamma_{1}+2, \ldots, \gamma_{1}+\gamma_{2}+1
$$




\section{The convergence of the MGOPT method}

The convergence of the CSMG scheme is already well established using local Fourier analysis; see for example [4,5]. Much less is known on the convergence properties of the MGOPT scheme that we discuss in the following. Assume that $\hat{J}_{k}\left(u_{k}\right)-\left(f_{k}, u_{k}\right)_{k}$ is twice Frechét differentiable for each $k$. Moreover, $\nabla^{2} \hat{J}_{k}$ is (locally) positive definite and satisfies the following conditions: $\left(\nabla^{2} \hat{J}_{k}(u) v, v\right)_{k} \geq \beta\|v\|_{k}^{2}$ and $\left\|\nabla^{2} \hat{J}_{k}(u)-\nabla^{2} \hat{J}_{k}(v)\right\|_{k} \leq \lambda\|u-v\|_{k}$ uniformly for some constants $\beta, \lambda>0$. We use the expansion

$$
\hat{J}_{k}(u+z)=\hat{J}_{k}(u)+\left(f_{k}, z\right)+\left(\nabla \hat{J}_{k}(u)-f_{k}, z\right)_{k}+\frac{1}{2} \int_{0}^{1}\left(\nabla^{2} \hat{J}_{k}(u+t z) z, z\right)_{k} d t .
$$

The following lemma will be necessary for the results that follow.

Lemma 5 Assume that $\left(\nabla \hat{J}_{k}(u)-f_{k}, v\right)_{k} \leq 0$ for $u, v \in V_{k}$. Let $\gamma$ satisfies the condition

$$
0 \leq \gamma \leq-2 \delta\left(\nabla \hat{J}_{k}(u)-f_{k}, v\right)_{k}\left[\int_{0}^{1}\left(\nabla^{2} \hat{J}_{k}(u+t \gamma v) v, v\right)_{k} d t\right]^{-1} \quad \text { for some } \delta \in[0,1] \text {. }
$$

Then

$-\gamma(1-\delta)\left(\nabla \hat{J}_{k}(u)-f_{k}, v\right)_{k} \leq \hat{J}_{k}(u)-\hat{J}_{k}(u+\gamma v)+\gamma\left(f_{k}, v\right) \leq-\gamma\left(\nabla \hat{J}_{k}(u)-f_{k}, v\right)_{k}$.

Proof. Set $z=\gamma v$ in Equation (37). For the first inequality, the restriction to $\gamma$ is used.

$$
\begin{aligned}
\hat{J}_{k}(u+\gamma v)= & \hat{J}_{k}(u)+\gamma\left(f_{k}, v\right)+\left(\nabla \hat{J}_{k}(u)-f_{k}, \gamma v\right)_{k}+\frac{1}{2} \int_{0}^{1}\left(\nabla^{2} \hat{J}_{k}(u+t \gamma v) \gamma v, \gamma v\right)_{k} d t \\
= & \hat{J}_{k}(u)+\gamma\left(f_{k}, v\right)+\gamma\left(\nabla \hat{J}_{k}(u)-f_{k}, v\right)_{k}+\frac{\gamma}{2} \int_{0}^{1}\left(\nabla^{2} \hat{J}_{k}(u+t \gamma v) v, v\right)_{k} d t \cdot \gamma \\
\leq & \hat{J}_{k}(u)+\gamma\left(f_{k}, v\right)+\gamma\left(\nabla \hat{J}_{k}(u)-f_{k}, v\right)_{k}+ \\
& \frac{\gamma}{2} \int_{0}^{1}\left(\nabla^{2} \hat{J}_{k}(u+t \gamma v) v, v\right)_{k} d t\left(-2 \delta\left(\nabla \hat{J}_{k}(u)-f_{k}, v\right)_{k}\left[\int_{0}^{1}\left(\nabla^{2} \hat{J}_{k}(u+t \gamma v) v, v\right)_{k} d t\right]^{-1}\right) \\
\leq & \hat{J}_{k}(u)+\gamma\left(f_{k}, v\right)+\gamma\left(\nabla \hat{J}_{k}(u)-f_{k}, v\right)_{k}-\gamma \delta\left(\nabla \hat{J}_{k}(u)-f_{k}, v\right)_{k} .
\end{aligned}
$$

Then

$$
-\gamma(1-\delta)\left(\nabla \hat{J}_{k}(u)-f_{k}, v\right)_{k} \leq \hat{J}_{k}(u)-\hat{J}_{k}(u+\gamma v)+\gamma\left(f_{k}, v\right)
$$

which proves the first inequality stated in the lemma. For the second inequality, the positivity of $\nabla^{2} \hat{J}_{k}$ is used. We have

$$
\begin{aligned}
\hat{J}_{k}(u+\gamma v) & =\hat{J}_{k}(u)+\gamma\left(f_{k}, v\right)+\left(\nabla \hat{J}_{k}(u)-f_{k}, \gamma v\right)_{k}+\frac{1}{2} \int_{0}^{1}\left(\nabla^{2} \hat{J}_{k}(u+t \gamma v) \gamma v, \gamma v\right)_{k} d t \\
& =\hat{J}_{k}(u)+\gamma\left(f_{k}, v\right)+\gamma\left(\nabla \hat{J}_{k}(u)-f_{k}, v\right)_{k}+\frac{\gamma^{2}}{2} \int_{0}^{1}\left(\nabla^{2} \hat{J}_{k}(u+t \gamma v) v, v\right)_{k} d t \\
& \geq \hat{J}_{k}(u)+\gamma\left(f_{k}, v\right)+\gamma\left(\nabla \hat{J}_{k}(u)-f_{k}, v\right)_{k} .
\end{aligned}
$$

Then

$$
\hat{J}_{k}(u)-\hat{J}_{k}(u+\gamma v)+\gamma\left(f_{k}, v\right) \leq-\gamma\left(\nabla \hat{J}_{k}(u)-f_{k}, v\right)_{k} .
$$

We now discuss an explicit estimate for the step length $\alpha$ such that the condition of sufficient decrease is satisfied. This is proven in the lemma that follows. 
Lemma 6 Assume that $\left(\nabla \hat{J}_{k}(u)-f_{k}, v\right)_{k} \leq 0$ for $u, v \in V_{k}$. Let

$$
\alpha(u, v)=\min \left\{2, \frac{-\left(\nabla \hat{J}_{k}(u)-f_{k}, v\right)_{k}}{\lambda\|v\|_{k}^{3}+\left(\nabla^{2} \hat{J}_{k}(u) v, v\right)_{k}}\right\} \quad \text { where } \lambda>0 .
$$

Then

$$
0 \leq-\frac{1}{2} \alpha(u, v)\left(\nabla \hat{J}_{k}(u)-f_{k}, v\right)_{k} \leq \hat{J}_{k}(u)-\hat{J}_{k}(u+\alpha(u, v) v)+\alpha(u, v)\left(f_{k}, v\right) .
$$

Proof. Set $\gamma=\alpha(u, v), \delta=\frac{1}{2}$ and $z=\alpha(u, v) v$ in Lemma 5. First, we will show that

$$
\begin{aligned}
\int_{0}^{1}\left(\nabla^{2} \hat{J}_{k}(u+t \alpha v) v, v\right)_{k} d t \leq \lambda\|v\|_{k}^{3}+\left(\nabla^{2} \hat{J}_{k}(u) v, v\right)_{k} . \\
\int_{0}^{1}\left(\nabla^{2} \hat{J}_{k}(u+t \alpha v) v, v\right)_{k} d t \\
=\int_{0}^{1}\left[\left(\nabla^{2} \hat{J}_{k}(u+t \alpha v) v, v\right)_{k}-\left(\nabla^{2} \hat{J}_{k}(u) v, v\right)_{k}+\left(\nabla^{2} \hat{J}_{k}(u) v, v\right)_{k}\right] d t \\
=\int_{0}^{1}\left[\left(\nabla^{2} \hat{J}_{k}(u+t \alpha v) v, v\right)_{k}-\left(\nabla^{2} \hat{J}_{k}(u) v, v\right)_{k}\right] d t+\int_{0}^{1}\left(\nabla^{2} \hat{J}_{k}(u) v, v\right)_{k} d t \\
=\int_{0}^{1}\left[\left(\nabla^{2} \hat{J}_{k}(u+t \alpha v) v, v\right)_{k}-\left(\nabla^{2} \hat{J}_{k}(u) v, v\right)_{k}\right] d t+\left(\nabla^{2} \hat{J}_{k}(u) v, v\right)_{k} \\
\leq \int_{0}^{1} \lambda\|t \alpha v\|_{k}\|v\|_{k}^{2} d t+\left(\nabla^{2} \hat{J}_{k}(u) v, v\right)_{k} \\
=\lambda \alpha\|v\|_{k}^{3} \int_{0}^{1} t d t+\left(\nabla^{2} \hat{J}_{k}(u) v, v\right)_{k} \\
\leq \lambda\|v\|_{k}^{3}+\left(\nabla^{2} \hat{J}_{k}(u) v, v\right)_{k}, \text { since } \alpha \leq 2 .
\end{aligned}
$$

Then from the given assumption for $\alpha$, we have

$$
\alpha(u, v) \leq \frac{-\left(\nabla \hat{J}_{k}(u)-f_{k}, v\right)_{k}}{\lambda\|v\|_{k}^{3}+\left(\nabla^{2} \hat{J}_{k}(u) v, v\right)_{k}} \leq \frac{-\left(\nabla \hat{J}_{k}(u)-f_{k}, v\right)_{k}}{\int_{0}^{1}\left(\nabla^{2} \hat{J}_{k}(u+t \alpha v) v, v\right)_{k} d t} .
$$

Notice that $\alpha$ satisfies the condition of Lemma 5 with $\delta=\frac{1}{2}$. Hence it follows that

$$
0 \leq-\frac{1}{2} \alpha(u, v)\left(\nabla \hat{J}_{k}(u)-f_{k}, v\right)_{k} \leq \hat{J}_{k}(u)-\hat{J}_{k}(u+\alpha(u, v) v)+\alpha(u, v)\left(f_{k}, v\right) .
$$

Finally, we will discuss the lemma which shows that the coarse-to-fine minimization with step length $\alpha$ given by Lemma 6 is a minimizing step. It is also not required to solve the coarse grid minimization problem exactly. This is however (formally) required on the coarsest grid.

Lemma 7 Let $u \in V_{k}$ and define $\tilde{u}=I_{k}^{k-1} u$. Let $\tilde{v} \in V_{k-1}$ and define $v=I_{k-1}^{k}(\tilde{v}-\tilde{u})$. Assume

$$
\hat{J}_{k-1}(\tilde{v})-\left(f_{k-1}, \tilde{v}\right) \leq \hat{J}_{k-1}(\tilde{u})-\left(f_{k-1}, \tilde{u}\right)
$$

where

$$
\left(f_{k-1}, \tilde{v}\right)=\left(I_{k}^{k-1} f_{k}+\nabla \hat{J}_{k-1}(\tilde{v})-I_{k}^{k-1} \nabla \hat{J}_{k}(v), \tilde{v}\right)
$$


and

$$
\left(f_{k-1}, \tilde{u}\right)=\left(I_{k}^{k-1} f_{k}+\nabla \hat{J}_{k-1}(\tilde{u})-I_{k}^{k-1} \nabla \hat{J}_{k}(u), \tilde{u}\right)
$$

respectively. Then

$$
\hat{J}_{k}(u+\alpha(u, v) v)-\hat{J}_{k}(u)-\alpha(u, v)\left(f_{k}, v\right) \leq \frac{1}{2} \alpha(u, v)\left(\nabla \hat{J}_{k}(u)-f_{k}, v\right)_{k}
$$

where $\alpha$ is the same as in Lemma 6. Strict inequality holds if

$$
\hat{J}_{k-1}(\tilde{v})-\left(f_{k-1}, \tilde{v}\right)<\hat{J}_{k-1}(\tilde{u})-\left(f_{k-1}, \tilde{u}\right) .
$$

Proof. First, show that $\left(\nabla \hat{J}_{k}(u)-f_{k}, v\right)_{k} \leq 0$. From Equation (37),

$$
\begin{aligned}
\left(\nabla \hat{J}_{k}(u)-f_{k}, z\right)_{k} & =\hat{J}_{k}(u+z)-\hat{J}_{k}(u)-\left(f_{k}, z\right)-\frac{1}{2} \int_{0}^{1}\left(\nabla^{2} \hat{J}_{k}(u+t z) z, z\right)_{k} d t \\
& \leq \hat{J}_{k}(u+z)-\hat{J}_{k}(u)-\left(f_{k}, z\right) .
\end{aligned}
$$

Let $z=\tilde{v}-\tilde{u}, u=\tilde{u}$ and replace $k$ by $k-1$. Then

$$
\begin{aligned}
\left(\nabla \hat{J}_{k-1}(\tilde{u})-f_{k-1}, \tilde{v}-\tilde{u}\right)_{k-1} & \leq \hat{J}_{k-1}(\tilde{v})-\hat{J}_{k-1}(\tilde{u})-\left(f_{k-1}, \tilde{v}-\tilde{u}\right) \\
& =\hat{J}_{k-1}(\tilde{v})-\left(f_{k-1}, \tilde{v}\right)-\left[\hat{J}_{k-1}(\tilde{u})-\left(f_{k-1}, \tilde{u}\right)\right] \leq 0 .
\end{aligned}
$$

Also, we have

$$
\begin{aligned}
\left(\nabla \hat{J}_{k}(u)-f_{k}, v\right)_{k} & =\left(\nabla \hat{J}_{k}(u)-f_{k}, I_{k-1}^{k}(\tilde{v}-\tilde{u})\right)_{k} \\
& =\left(I_{k}^{k-1}\left(\nabla \hat{J}_{k}(u)-f_{k}\right), \tilde{v}-\tilde{u}\right)_{k-1} \\
& =\left(\nabla \hat{J}_{k-1}(\tilde{u})-f_{k-1}, \tilde{v}-\tilde{u}\right)_{k-1} \leq 0 .
\end{aligned}
$$

The last equality can be referred to Remark 3 and it follows from Lemma 6 that

$$
\hat{J}_{k}(u+\alpha(u, v) v)-\hat{J}_{k}(u)-\alpha(u, v)\left(f_{k}, v\right) \leq \frac{1}{2} \alpha(u, v)\left(\nabla \hat{J}_{k}(u)-f_{k}, v\right)_{k} .
$$

It appears clearly from the proofs that global convergence of the MGOPT method depends critically on the descent direction $d$ which in turn requires local positivity of the Hessian. We can conclude with the following remarks.

Remark 8 For $d$ to be a descent direction the following three conditions should be satisfied [12].

- The convexity properties of the optimization problem on the finest resolution must be well approximated by the problem on the coarse resolution .

- The multigrid subproblems $\min _{x_{k-1}}\left(\hat{J}_{k-1}\left(u_{k-1}\right)-\left(f_{k-1}, u_{k-1}\right)_{k-1}\right)$ are solved with certain accuracy.

- The restriction and prolongation operators satisfy the condition

$$
I_{k-1}^{k}=c\left(I_{k}^{k-1}\right)^{\top} \quad \text { for a constant } c>0 .
$$

This assumption is standard for multigrid algorithms. In our case, this is true for $I_{k}^{k-1}$ as a full weighing restriction and $I_{k-1}^{k}$ as a bilinear interpolation with $c=4$. 
We state the convergence of the MGOPT method.

Theorem 9 Let $\hat{J}_{k}\left(u_{k}\right)-\left(f_{k}, u_{k}\right)_{k}$ be twice Frechét differentiable for each $k$. Moreover, let $\nabla^{2} \hat{J}_{k}$ be locally Lipschitz continuous and satisfies $\left(\nabla^{2} \hat{J}_{k}(u) v, v\right)_{k} \geq \beta\|v\|_{k}^{2}$ together with $\left\|\nabla^{2} \hat{J}_{k}(u)-\nabla^{2} \hat{J}_{k}(v)\right\|_{k} \leq \lambda\|u-v\|_{k}$ uniformly for some positive constants $\beta$ and $\lambda$ in a neighborhood $V_{k}^{\epsilon}$ of $u_{k}^{*}$ where $u_{k}^{*}$ is the solution to problem (28). Then MGOPT method provides a minimizing iteration.

Proof. Let $u_{k}^{0} \in V_{k}^{\epsilon}$. Then $A=\left\{u \in V_{k}: \hat{J}_{k}(u)-\left(f_{k}, u\right) \leq \hat{J}_{k}\left(u_{k}^{0}\right)-\left(f_{k}, u_{k}^{0}\right)\right\}$ is a compact set. By induction, let $k=2$ and define $u_{k}$ to be the result of the MGOPT step and $\hat{u}_{k-1}=\arg \min _{\mathrm{u} \in \mathrm{V}_{\mathrm{k}-1}}\left(\hat{J}_{k-1}(u)-\left(f_{k-1}, u\right)_{k-1}\right)$. Using the result of Lemma 7, we have

$$
\begin{aligned}
\hat{J}_{k}\left(u_{k}\right)-\left(f_{k}, u_{k}\right)_{k} & =\hat{J}_{k}\left(S_{k}^{\gamma_{2}}\left(u_{k}^{\gamma_{1}+1}\right)\right)-\left(f_{k}, S_{k}^{\gamma_{2}}\left(u_{k}^{\gamma_{1}+1}\right)\right) \\
& \left.\leq \hat{J}_{k}\left(u_{k}^{\gamma_{1}}+\alpha I_{k-1}^{k}\left(\hat{u}_{k-1}-I_{k}^{k-1} u_{k}^{\gamma_{1}}\right)\right)-\left(f_{k}, u_{k}^{\gamma_{1}}+\alpha I_{k-1}^{k}\left(\hat{u}_{k-1}-I_{k}^{k-1} u_{k}^{\gamma_{1}}\right)\right)\right) \\
& \leq \hat{J}_{k}\left(u_{k}^{\gamma_{1}}\right)-\left(f_{k}, u_{k}^{\gamma_{1}}\right)_{k} \\
& =\hat{J}_{k}\left(S_{k}^{\gamma_{1}}\left(u_{k}^{0}\right)\right)-\left(f_{k}, S_{k}^{\gamma_{1}}\left(u_{k}^{0}\right)\right)_{k} \\
& \leq \hat{J}_{k}\left(u_{k}^{0}\right)-\left(f_{k}, u_{k}^{0}\right)_{k} .
\end{aligned}
$$

Note that strict inequality holds in all steps if $\nabla \hat{J}_{k}-f_{k}$ is nonzero. For $k>2$, by induction hypothesis and Lemma 7, the theorem holds.

\section{Numerical results}

In this section, we present the results of numerical experiments on the computational performance of the proposed multigrid schemes as solvers for distributed elliptic optimal control problems. Choosing different values of the weight of the cost of the control, we gathered the CPU time (in seconds) until a stopping tolerance of $t o l=10^{-8}$ for the norm of the reduced gradient is satisfied. For all computations, we use $\gamma_{1}=\gamma_{2}=2$ pre and post smoothing steps. This means that one cycle of the CSMG and of the MGOPT method uses $\gamma_{1}+\gamma_{2}=4$ iterations of the smoothing algorithm on the finest grid. We consider an elliptic optimal control problem with a discontinuous target function $z$ as depicted in Figure 1. We use this setting when solving both the linear and bilinear control problems.

First, we discuss the following linear elliptic optimal control problem

$$
\left\{\begin{aligned}
\min _{u \in U} J(y, u):=\frac{1}{2}\|y-z\|_{L^{2}}^{2}+\frac{\nu}{2}\|u\|_{L^{2}}^{2}, \\
-\Delta y-u=f \text { in } \Omega, \\
y=0 \text { on } \partial \Omega .
\end{aligned}\right.
$$

Let $\Omega=(0,1) \times(0,1)$ and $f, z \in L^{2}(\Omega)$ given by

$$
\begin{aligned}
& f(x, y)=1 \\
& z(x, y)=\left\{\begin{array}{ll}
2, & \text { on }(0.25,0.75) \times(0.25,0.75) \\
1, & \text { otherwise }
\end{array} .\right.
\end{aligned}
$$

Notice that $z$ is not attainable because of the boundary conditions. The numerical results for this case are shown in Tables 1 and 2. We can see from Table 1 that choosing different values for the parameter $\nu$, the CSMG method converges within eight iterations. We also obtain that the number of iterations is independent on the parameter $\nu$ and on the mesh size. For the one grid optimization scheme, we use the gradient method (GM) and the nonlinear 


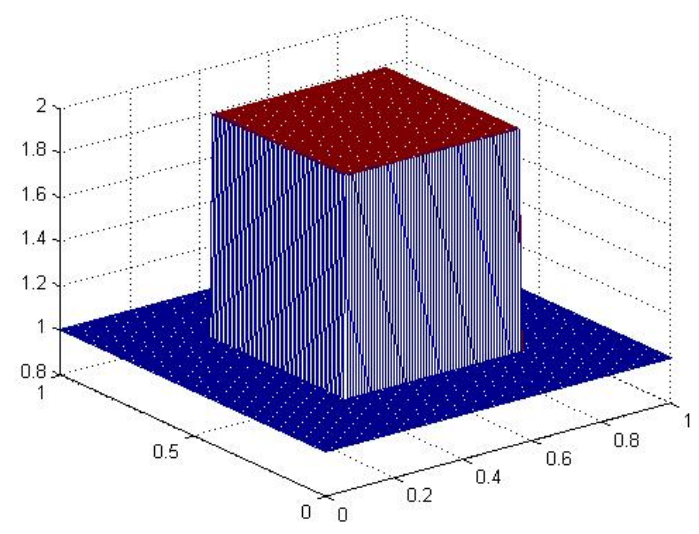

Figure 1: The target function $z$.

conjugate gradient (NCG) scheme [13]. As shown in Table 2, there is a constant increase in the computational time as the parameter $\nu$ decreases. This property is also inherited by the MGOPT method using both GM and NCG as smoothing algorithms. MGOPT with NCG on the other hand exhibits a faster rate of convergence compared to the MGOPT with GM. In all cases, the MGOPT procedure greatly improves upon the stand-alone one grid optimization scheme and the resulting performance may become comparable to the optimal performance of the CSMG algorithm. The numerical solutions $y$ and $u$ for $\nu=10^{-4}$ are shown in Figure 2.

Table 1: Results of linear elliptic optimal control problem using CSMG method.

\begin{tabular}{ccccccr}
\hline$\nu$ & mesh & iter & $\hat{J}$ & $\|\nabla \hat{J}\|_{L_{2}}$ & $\rho$ & time $(\mathrm{sec})$ \\
\hline \hline & $65 \times 65$ & 8 & 0.718 & $9.64 \mathrm{e}-11$ & 0.053 & 0.8 \\
$1 \mathrm{e}-2$ & $129 \times 129$ & 8 & 0.701 & $9.91 \mathrm{e}-11$ & 0.053 & 3.2 \\
& $257 \times 257$ & 8 & 0.692 & $9.95 \mathrm{e}-11$ & 0.054 & 15.3 \\
\hline & $65 \times 65$ & 8 & 0.163 & $3.96 \mathrm{e}-12$ & 0.090 & 0.8 \\
$1 \mathrm{e}-4$ & $129 \times 129$ & 8 & 0.154 & $3.90 \mathrm{e}-12$ & 0.083 & 3.2 \\
& $257 \times 257$ & 8 & 0.151 & $3.88 \mathrm{e}-12$ & 0.082 & 15.3 \\
\hline
\end{tabular}

Next, we discuss a bilinear elliptic optimal control problem as follows

$$
\left\{\begin{aligned}
\min _{u \in U} J(y, u):=\frac{1}{2}\|y-z\|_{L^{2}}^{2}+\frac{\nu}{2}\|u\|_{L^{2}}^{2}, \\
-\Delta y-u y=f \text { in } \Omega, \\
y=0 \text { on } \partial \Omega .
\end{aligned}\right.
$$

Let $\Omega=(0,1) \times(0,1)$ and $f, z \in L^{2}(\Omega)$ given by

$$
\begin{aligned}
& f(x, y)=1 \\
& z(x, y)= \begin{cases}2, & \text { on }(0.25,0.75) \times(0.25,0.75) \\
1, & \text { otherwise }\end{cases}
\end{aligned}
$$

For this case numerical results are shown in Tables 3 and 4 . We can see from Table 3 that the CSMG method exhibits almost independence of the number of iterations on $\nu$ on the size 
Table 2: Results of CPU time (seconds) of linear elliptic optimal control problem using gradient method (GM), MGOPT with GM (MGOPT ${ }^{1}$ ), nonlinear conjugate gradient (NCG) and MGOPT with NCG $\left(\mathrm{MGOPT}^{2}\right)$.

\begin{tabular}{ccrrrr}
\hline$\nu$ & mesh & GM & MGOPT $^{1}$ & NCG & MGOPT $^{2}$ \\
\hline \hline & $65 \times 65$ & 2.2 & 1.9 & 0.9 & 0.9 \\
$1 \mathrm{e}-2$ & $129 \times 129$ & 11.3 & 8.7 & 4.5 & 4.1 \\
& $257 \times 257$ & 57.5 & 40.0 & 23.1 & 20.7 \\
\hline \multirow{3}{*}{$1 \mathrm{e}-4$} & $65 \times 65$ & 27.9 & 23.0 & 4.9 & 4.1 \\
& $129 \times 129$ & 144.1 & 74.3 & 26.2 & 23.2 \\
& $257 \times 257$ & 723.0 & 344.8 & 131.8 & 88.6 \\
\hline
\end{tabular}

Figure 2: Numerical solutions for the state (left) and control (right) variables of the linear elliptic optimal control problem using $\nu=10^{-4}$.
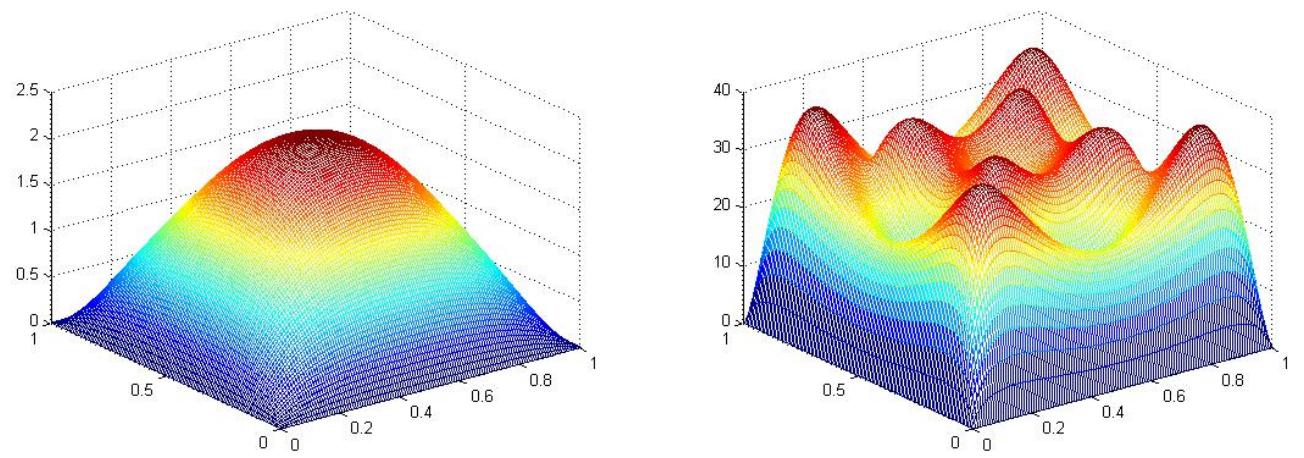

of the mesh where the problem is being solved. Together with the total CPU time, we also include the CPU time for the computation of the roots of the quartic polynomial as this takes about $70 \%$ of the whole computational time. Here, our purpose is to show robustness of the collective smoothing procedure while we have made no effort in implementing a fast quartic polynomial solver. Similar to the linear case, we show in Table 4 the computational time for the onegrid optimization scheme using GM and NCG schemes. For solution processes exceeding 20,000 seconds we stopped the calculation. This table shows that MGOPT with NCG is faster than MGOPT with GM. The numerical solutions $y$ and $u$ for $\nu=10^{-4}$ are shown in Figure 3.

\section{Conclusion}

We presented two multigrid schemes for solving elliptic optimal control problems, the CSMG and the MGOPT methods. The numerical results showed that the CSMG scheme is faster compared to the MGOPT method. It also provides mesh independent and parameter independent convergence. On the other hand, it was shown that the MGOPT scheme greatly accelerates one grid optimization schemes. While the application of the CSMG scheme required a carefully designed collective smoother specific for each problem, the application of the MGOPT scheme did not require any adaptation to the problem. 
Table 3: Results of bilinear elliptic optimal control problem using CSMG method. (* time for computing the roots)

\begin{tabular}{ccccccrr}
\hline & mesh & iter & $\hat{J}$ & $\|\nabla \hat{J}\|_{L_{2}}$ & $\rho$ & time $(\mathrm{sec})$ & time $(\mathrm{sec})$ \\
\hline \hline \multirow{3}{*}{$1 \mathrm{e}-2$} & $65 \times 65$ & 8 & 0.863 & $2.06 \mathrm{e}-11$ & 0.081 & 47.6 & 33.2 \\
& $129 \times 129$ & 9 & 0.843 & $1.85 \mathrm{e}-12$ & 0.083 & 221.9 & 155.8 \\
& $257 \times 257$ & 9 & 0.834 & $1.90 \mathrm{e}-12$ & 0.084 & 912.8 & 641.3 \\
\hline \multirow{3}{*}{$1 \mathrm{e}-4$} & $65 \times 65$ & 10 & 0.158 & $1.55 \mathrm{e}-08$ & 0.287 & 59.7 & 41.5 \\
& $129 \times 129$ & 10 & 0.151 & $1.46 \mathrm{e}-08$ & 0.366 & 243.1 & 170.1 \\
& $257 \times 257$ & 10 & 0.148 & $1.45 \mathrm{e}-08$ & 0.434 & 997.5 & 696.7 \\
\hline
\end{tabular}

Table 4: Results of CPU time (seconds) of bilinear elliptic optimal control problem using gradient method (GM), MGOPT with GM (MGOPT ${ }^{1}$ ), nonlinear conjugate gradient (NCG) and MGOPT with NCG $\left(\mathrm{MGOPT}^{2}\right)$. (- longer that 20,000 seconds)

\begin{tabular}{ccrrrr}
\hline \multicolumn{1}{c}{ mesh } & GM & MGOPT $^{1}$ & NCG & MGOPT $^{2}$ \\
\hline \hline & $65 \times 65$ & 1.2 & 1.0 & 0.8 & 0.8 \\
$1 \mathrm{e}-2$ & $129 \times 129$ & 5.2 & 4.9 & 4.0 & 3.7 \\
& $257 \times 257$ & 25.1 & 19.1 & 19.0 & 18.8 \\
\hline & $65 \times 65$ & - & - & 17079.0 & 160.7 \\
$1 \mathrm{e}-4$ & $129 \times 129$ & - & - & - & 805.7 \\
& $257 \times 257$ & - & - & - & 4112.4 \\
\hline
\end{tabular}

Figure 3: Numerical solutions for the state (left) and control (right) variables of the bilinear elliptic optimal control problem using $\nu=10^{-4}$.
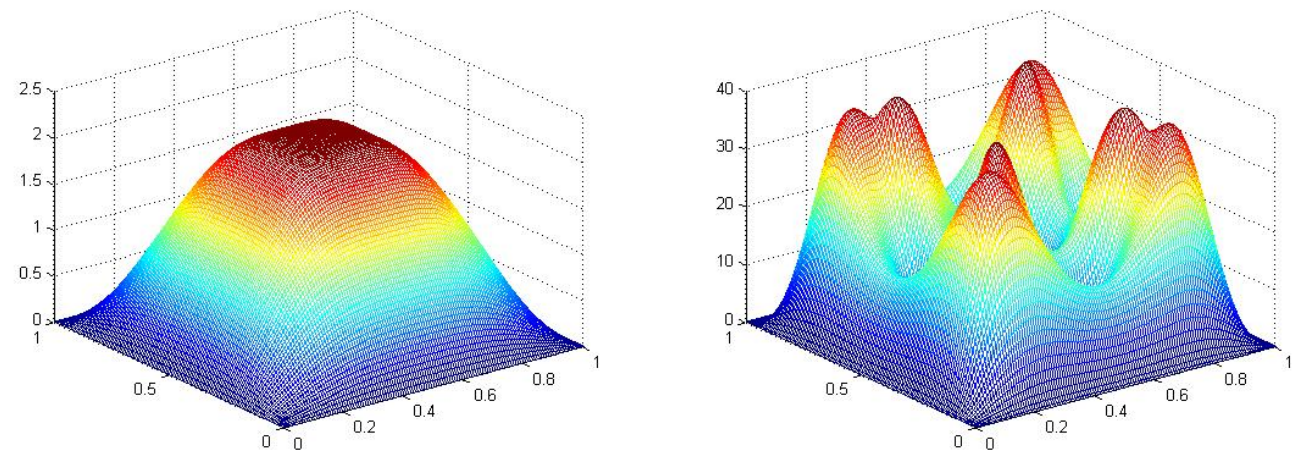

\section{Acknowledgement}

Special thanks to Stephen Nash and Craig C. Douglas for stimulating discussions and support. 


\section{References}

[1] A. Bonzì, Multigrid methods for parabolic distributed optimal control problems, Journal of Computational and Applied Mathematics, 157 (2003), pp. 365-382.

[2] A. BORZÌ, A multigrid scheme for elliptic constrained optimal control problems, Computational Optimization and Applications, 31 (3) (2005), pp. 309-333.

[3] A. BoRZì, On the convergence of the MG/OPT method, PAMM, 5 (1) (2005), pp. 735-736.

[4] A. BORZÌ, High-order discretization and multigrid solution of elliptic nonlinear constrained optimal control problems, Journal of Computational and Applied Mathematics, 200 (1) (2007), pp. 67-85.

[5] A. BORZİ, K. KUNISCH, AND D. KWAK, accuracy and convergence properties of the finite difference multigrid solution of an optimal control optimality system, SIAM Journal on Control and Optimization, 41 (5) (2003), pp. 1477-1497.

[6] A. BRAnDT, Multi-level adaptive solutions to boundary-value problems, Mathematics of Computation, 31 (1977), pp. 333-390.

[7] V. GEORGESCU, On the unique continuation property for Schrödinger Hamiltonians, Helv. Phys. Acta, 52 (1979), pp. 655-670.

[8] M.D. Gunzburger, L. Hou, And T.P. Svobodny, Finite element approximations of an optimal control problem associated with the scalar Ginzburg-Landau equation, Comput. Math. Appl., 21 (1991), pp. 123-131.

[9] R. M. LEWIS AND S. G. NASH, A multigrid approach to the optimization of systems governed by differential equations, AIAA-2000-4890, 8th AIAA/USAF/NASA/ISSMO Symposium on Multidisciplinary Analysis and Optimization, Long Beach, CA, (2000).

[10] R. M. LEWIS AND S. G. NASH, Model problems for the multigrid optimization of systems governed by differential equations, SIAM Journal on Scientific Computing, 26 (6) (2005), pp. 1811-1837.

[11] J.L. Lions, Optimal Control of Systems Governed by Partial Differential Equations, Springer, Berlin, 1971.

[12] S. G. NASH, A multigrid approach to discretized optimization problems, Optimization Methods and Software, 14 (1 \& 2) (2000), pp. 99-116.

[13] J. Nocedal, AND S.J. Wright, Numerical Optimization, Springer-Verlag, New York, 1999.

[14] S. OH, C. Bouman, AND K. WeBB, Multigrid tomographic inversion with variable resolution data and image spaces, IEEE Transactions on Image Processing, 15 (9) (2006), pp. 2805-2819.

[15] S. OH, A. Milstein, C. Bouman, AND K. WeBb, A general framework for nonlinear multigrid inversion, IEEE Transactions on Image Processing, 14 (1)(2005), pp. 125-140. 
[16] S. OH, A. Milstein, C. Bouman, And K. WebB, Multigrid algorithms for optimization and inverse problems, IEEE Transactions on Image Processing, 14 (1) (2005), pp. 125-140.

[17] J.C. Ye, C. Bouman, K. Webb, And R. Millane, Nonlinear multigrid algorithms for bayesian optical diffusion tomography, IEEE Transactions on Image Processing, 10 (6) (2001), pp. 909-922. 